\title{
Scaffolding Collaborative Learning Opportunities: Integrating Microworld Use and Argumentation
}

\author{
Toby Dragon ${ }^{1}$, Bruce M. McLaren ${ }^{1,2}$, Manolis Mavrikis ${ }^{3}$, Eirini Geraniou ${ }^{4}$ \\ ${ }^{1}$ CeLTech, Saarland University, Germany, ${ }^{2}$ Carnegie Mellon University, USA \\ ${ }^{3}$ London Knowledge Lab, Institute of Education, University of London, UK, ${ }^{4}$ School of \\ Education, University of Southampton, UK \\ toby.dragon@celtch.de,bmclaren@cs.cmu.edu,m.mavrikis@lkl.ac.uk, \\ e.geraniou@soton.ac.uk
}

\begin{abstract}
This paper presents our research efforts to support students' collaborative process when learning mathematics and science as they interact in microworlds and engage in discussions and structured arguments. From a pedagogical perspective, the system provides students with an environment to explore challenging problems and encourages them to collaborate. The collaboration takes place in a discussion environment that is integrated with microworlds, allowing students to discuss and argue with one another and share their rationales and insights. The challenge of this work lies in providing students, teachers, and researchers with coherent, unified feedback within the system as a whole. To accomplish this, the system must combine and analyze student actions across tools, and results of those actions. We conclude that the integration of these two types of software tools provides a solid foundation for intelligent analysis of student collaboration.
\end{abstract}

Keywords: Collaboration, intelligent support, microworlds, argumentation, discussion

\section{Introduction}

Technological advances and research in technology-enhanced learning (TEL) have enabled at least two ways in which computer-based environments can support the way students learn mathematics and science. The first is through Exploratory Learning Environments (ELEs) including microworlds and simulations, which hold the promise of making abstract ideas concrete and manipulable $[1,2]$. The second is through computer supported collaborative learning (CSCL) and particularly dialogue and argumentation $[3,4,5]$ which provide students the means to engage in discussions and structure arguments.

The work presented here attempts to blend these two approaches to learning by integrating ELEs with a discussion and argumentation environment, thus enabling the possibility to learn in ways that were not previously possible. Some prior steps have been taken in this direction; for instance, the CoChemEx project explored the combined use of a virtual laboratory environment with a collaborative discussion 
environment, finding that scripted use of the integrated environment was easier for students than a non-scripted environment [6]. The Rashi project also experimented with combining tools for data exploration and argument construction in a collaborative context, finding that the addition of collaboration increased the amount of student effort within the system [7].

Integration of discussion and exploratory learning environments has the potential to provide unique learning opportunities. Students can support each other by sharing domain knowledge (a form of peer tutoring), and students arguing about their work can promote deeper understanding than the students could gain working independently. However, there is a large potential for confusion, or missed opportunity when students are working in different tools and with different conceptual knowledge. The unique aspect of this work is our attempt to use an intelligent support system to recognize differences in student's knowledge, and to support the movement between different tools in such a way that students gain the benefits of peer support and argumentation about constructed knowledge.

This work is being done within the context of an EU-funded project (Metafora ${ }^{1}$ ), which aims to provide a holistic environment in which students will collaboratively plan and organize their work, as well as collaborate in solving challenges and problems over a relatively long time period.

This paper presents a particular use case in mathematics and introduces the challenges that we face in our efforts to analyze students' collaborative process while they interact in a mathematical microworld and simultaneously have the opportunity to engage in discussions and structured arguments. In the microworld, called eXpresser, students construct patterns of repeated building blocks of square tiles and their associated algebraic rules, as described in more detail in the next section. Underlying this goal, the main objective is to promote students' appreciation of the power of algebra $[8,9] .{ }^{2}$ In parallel, students engage in discussions in $\mathrm{LASAD}^{3}$, a web-based argumentation tool that enables groups of learners to discuss their work in a structured way $[10,11]$. LASAD is a collaborative, shared workspace containing a graphical argumentation environment and a chat tool. Students use this space to share ideas and organize their thoughts as they learn new concepts, and discuss or argue.

Both of these tools have analysis agents that can provide intelligent support. Several computational components analyze students' interaction in eXpresser and a rule-based system offers suggestions or hints designed to help them complete the task they are undertaking [12]. The LASAD tool offers a generic framework for feedback [11] and a rule-based system that offers advice on the structure of arguments, such as whether "claims" are supported by "facts" and "questions" are answered with "answer" objects. The output from these analyses can be combined to offer feedback that supports collaboration and helps students make progress while they grapple with the challenge.

The Metafora system incorporates these tools (as well as other tools not mentioned here), providing communication and control abilities across tools. The tools, and their associated intelligent support components, are linked both through interface elements

\footnotetext{
${ }^{1} \mathrm{http}: / /$ www.metafora-project.org

${ }^{2}$ eXpresser was developed in the context of the MiGen project (see http://www.migen.org)

${ }^{3} \mathrm{http}: / /$ cscwlab.in.tu-clausthal.de/lasad/
} 
and data sharing components. Each tool records lower-level events (termed indicators) that note instances or summary of student activity, and higher-level events (termed landmarks) that note a significant accomplishment or evaluation of student work. An overall analysis component analyses these events to identify situations where intervention might encourage peer support or shared knowledge evaluation. To concretize the purpose, architecture, and usage of the system, section 2 presents a specific use case to illustrate how students might work within the system, and how the system might respond. Section 3 discusses our generic cross-tool analysis approach and section 4 concludes that this approach of integration and analysis across tools provides a solid foundation for supporting student collaborative process.

\section{The Integrated Microworld and Discussion Environment in Use}

This scenario is meant to highlight the potential benefits and challenges of integrating microworld and argumentation tools in a pedagogically meaningful way. We seek to demonstrate how analysis from the individual tools can be combined to recognize when students should be prompted to use a specific tool, and how they might be prompted to do so.

The challenge given to students in this scenario is to use eXpresser to derive algebraic rules that correspond to structures of their own design, and are general across variable values. Specifically, in eXpresser, students construct their own models made of square tiles. These models contain variables that can be changed dynamically to test their structural generality. For example, Fig. 1 shows a student's construction of a model that is comprised of two patterns, the red and the green. The red pattern (made of a building block of 2 tiles) is repeated horizontally 5 times. In an effort to make the model general and animate it, the student specifies that the green pattern (made of a building block of 5 tiles) is repeated 'one more time' than the red building block. To achieve this, the student creates a variable called 'gaps' to represent the number of gaps in the model. In order to color the model, the student has to specify algebraic expressions that represent the number of tiles in each pattern and subsequently define the model rule that represents the total number of tiles in the model. It is evident that the same model can be constructed in different ways, leading to different model rules. The description of the task and the classroom culture encourages students to construct structurally different models.

Subsequently, a collaborative task encourages students to discuss the correctness and equivalence (or not) of their derived rules. It challenges students to read, deconstruct and match their rule with their own model as well as with their partner's model. In previous work we have established the benefit of these collaborative tasks in that they provide students with opportunities to reflect on their interaction with the system and develop strategies that allow them to justify the correctness and equivalence of their rules [13]. We now envision that students are given this task within the Metafora system, which provides access to both the eXpresser and LASAD tools. The students can use LASAD to share and discuss their models with the other students in a group. Ultimately, the goal is for the students to reach an agreement and understanding of the importance and usage of algebraic rules. 


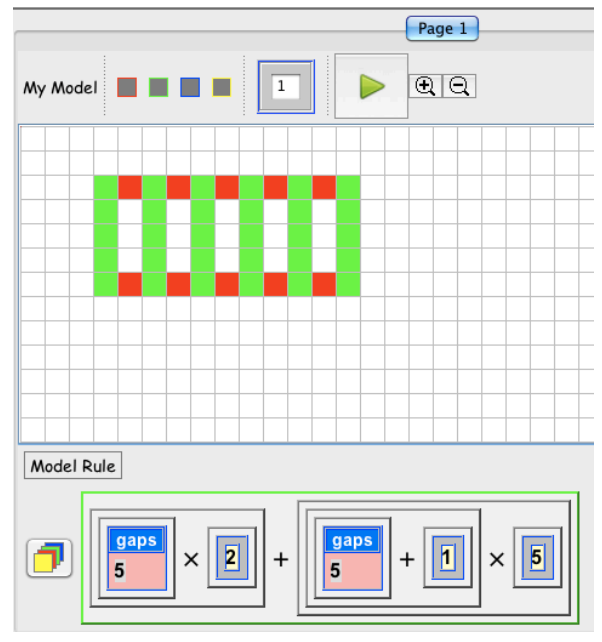

Fig. 1. A student's construction in eXpresser and its rule: a quasi-algebraic representation of the total number of tiles in the model.

As students are working individually at the beginning, the analytic tools of eXpresser look for landmarks, i.e. significant points that demonstrate important information about a student. One situation that might occur is that Student A achieves the landmark of creating a "general rule." The analytic tools of eXpresser recognize and report this event to the analysis channel in the Metafora framework. The Metafora analysis agent recognizes that Student B has not yet reached this landmark. The LASAD tool reports any sharing of models over the analysis channel as well, so the system can recognize that the two students have not discussed this model. Thus, we have a situation where Student A has reached an understanding that could be helpful if shared with Student B. If the system took no action, Student B might struggle a bit in doing her own generalization, or Students A and B could potentially discuss their findings and share knowledge on their own. However, if Student B continues to struggle, and Student A doesn't communicate her model with Student B, the system could suggest to Student A that she share her work with Student B and they discuss. Additionally, or alternatively, this information can be conveyed to the teacher who can take appropriate decisions.

Here we see some of the pedagogical benefits of linking the individual workspace with a group discussion space. Rather than relying entirely on automated feedback from within the microworld, we can exploit the advantages of collaboration to encourage students to help each other. Similar tactics for encouraging students to help one another have been suggested in prior work on the Rashi project where the system used an expert knowledge base to recognize differences between student knowledge and would then elicit conversation about these differences [14]. Likewise, in the Metafora system, we can recognize differences in landmarks for students, and encourage discussion in this context. 
The Metafora analysis agent then monitors indicators (the lower level events such as messages sent and statements created in the argument space) logged from the LASAD tool to the analysis channel of the Metafora framework. When the analysis agent recognizes that the model has been shared, and that a sufficient amount of conversation has occurred, the system suggests to Student B that she re-visit her model with the aim of reaching this landmark with her own solution. As the analysis system for LASAD matures for this specific application, LASAD itself could offer landmarks, such as recognition that the students have "shared knowledge", or "reached agreement". This is a challenge to be addressed, and can refer to earlier work in the ARGUNAUT project in which graph and text matching techniques were used to identify certain critical exchanges between students [15]).

Finally, after some time passes in which both students are moving between discussion and microworld environments, the analysis agent in the eXpresser system reports that student $\mathrm{B}$ has created her own general model, attaining the same landmark as originally attained by student A. Since the analysis agent is now aware that both students have achieved the landmark "general rule", the system refers both students to the discussion environment (if they are not both there), and prompts them to discuss questions like "How are your models different?", or "Convince each other that your models are correct?". Fig. 2 shows an example of the discussion that follows in LASAD. Both students provide arguments that, in their opinion, justify the correctness of their rule. However, in Student A's opinion, Student's B argument (Box 4) does not explain clearly why the rule is correct. Having been challenged, Student B provides a further explanation (Box 12) that demonstrates a better understanding of the microworld affordances and a growing appreciation of some algebraic concepts (e.g. by writing "even if the number changes the rule is always correct").

The LASAD analysis agent can analyze this discussion of differences and correctness, looking for patterns such as "lack of consensus". Again, here we see the benefit of combined systems, knowing that both students have reached the landmark (creating a "general" rule) allows the system to predict that they should reach consensus on the correctness of each model. We also see the major challenges offered by such a task, in recognizing a lack of consensus. The LASAD feedback agent employs rule-based pattern recognition using information such as the types of boxes used (e.g. claim, argument, explanation) and linkage between them, as well as limited text analysis (keywords, etc.) in an attempt to recognize patterns of argumentation. Once user data has been collected, this work can be extended using proven machinelearning techniques applied to similar discussion environments [15]. At least initially, the system is not likely to be precise enough in this type of decision to directly prompt interaction with students; rather, a message to a moderating teacher could be used to prompt her to offer advice and support. For example, if the system recognizes a "lack of consensus" on the correctness of a model that eXpresser has reported as being correct in a landmark, the system can report this situation to the teacher. If the teacher agrees with the diagnosis, or finds the situation interesting in any significant way, she can then intervene in the discussion helping students appreciate what has been preventing them from reaching consensus and promoting more effective collaboration. 


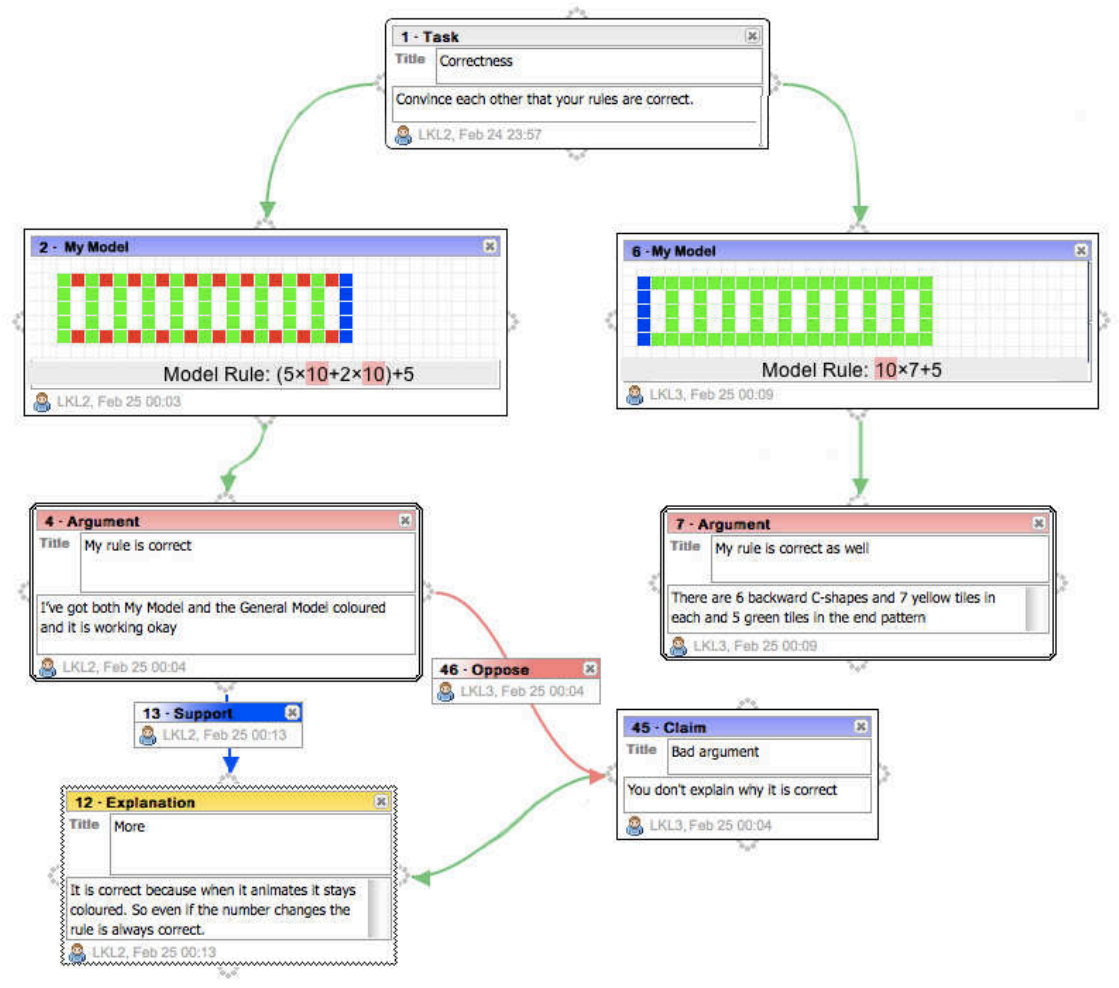

Fig. 2. An example of a LASAD discussion where students discuss the correctness of their eXpresser models.

\section{Generic Cross-Tool Analysis}

We have described a scenario in which an integrated microworld-discussion system could potentially provide great benefit to collaborating students. The integrated system can use the accumulated data across the tools to determine, for instance, when one student or another has reached a landmark. This can be a cue for the successful student to help the other student.

We now discuss our initial ideas on creating a system that can handle the abovementioned situation in a generic manner, working also in the context of different challenges, with different kinds of microworlds, and potentially different types of discussion environments, in a standardized manner. In this way, we describe how the analysis agent for the overall Metafora system can recognize and take action to create the scenario described above.

First, the intelligent analysis components of the separate tools must share information, in particular analysis and abstraction of student actions, which allows for 
unified analysis of the integrated learning system. The systems remain highly separated, with each individual tool running from its own server. The over-arching Metafora system maintains multiple communication channels for the interaction between tools: an analysis channel where tools' analysis components can report indicators and landmarks; and a command channel, where the system can instruct tools to display specific states or offer feedback to a specific end-user. The server logs and analyzes data coming in from the analysis channel, and provides commands to the tools based on this analysis.

Each tool reports processed information about the current users to the Metafora system (indicators and landmarks), and receives feedback information from the system to be presented to a user or a group of users. The challenge for the analysis agent on the Metafora server is to decide what is relevant information for the given task and tools. In the example above, we see that one relevant piece of information from the microworld is the generation of a landmark, in this case the accomplishment of the high-level task "creating a general rule". The discussion environment can provide other pertinent pieces of information by generating indicators of student activity: in this case indicators showing discussion of the artifacts involved in this landmark (e.g. references to the "general rule" model that have been shared in discussion). Considering the generated landmark, we can allow it to act as a phase judgment consisting of three phases, as presented in Table 1. Here we see that the landmark defined by the microworld helps define when and how the system encourages students to use the discussion tool.

Table 1. Cross-tool feedback. The system will encourage a certain behavior, according to the given landmark, and the tool in which students are currently engaged.

\begin{tabular}{|c|c|c|}
\hline $\begin{array}{c}\text { Landmark has been } \\
\text { Noted For... }\end{array}$ & Feedback in Microworld & Feedback in Discussion \\
\hline Neither student & $\begin{array}{c}\text { Provide students microworld- } \\
\text { specific feedback }\end{array}$ & $\begin{array}{c}\text { Prompt students to use the } \\
\text { microworld to explore task }\end{array}$ \\
\hline Only Student A & Prompt students to discuss Student A's microworld state \\
\hline Both Students & $\begin{array}{c}\text { Prompt a discussion of } \\
\text { differences between solutions }\end{array}$ & $\begin{array}{c}\text { Provide students discussion- } \\
\text { specific feedback }\end{array}$ \\
\hline
\end{tabular}

\section{Conclusions}

We propose that the combined analysis of individual activities (individual students' actions in a microworld) and collaborative activities (discussion of the microworld activities between students) can lead to productive intelligent support. The information provided by individual components can be used to define phases of work and recognize opportunities for productive collaboration. One major challenge of employing the approach described here is to generalize beyond the specific use case above. We have offered insight for a specific scenario between two tools and two 
users. Table 1 is the beginnings of a generic way of considering the state of individual tools in a more global way by the Metafora system. Future work includes scaling this type of support over multiple tools, in particular, to encompass different microworlds, and larger groups of students. Another major challenge, related to the first, is defining an abstraction layer that is able to capture and represent a variety of indicators and landmarks. Furthermore, such an abstraction layer must represent the connections between landmarks. For instance, in the example provided, there is a need for the landmark achieved by Student A to be linked to the need of a similar landmark for Student B.

With this effort, we also suggest a path that fellow researchers might follow in attempting to introduce collaborative activities into their current systems, or combine current systems to create collaborative workspaces. We suggest that single-user environments can be integrated with collaborative workspaces by adding small components to communicate student state information with external systems. We also demonstrate how current intelligent feedback agents can be integrated and extended to work with information across multiple tools by using simple message passing with a common language and data format. Such an approach can offer a solid foundation for taking many currently independent and specialized tools and creating a collaborative workspace that can offer holistic, intelligent support to students. Furthermore, such an approach can provide useful information to teachers and support them in their efforts to help students. Future work includes defining and implementing a teacher interface and interaction that will allow teachers to access and respond to such information, building off of previous similar efforts in the Argunaut and MiGen projects $[15,16]$.

Acknowledgments. We would like to thank the European Union under the Information and Communication Technologies (ICT) theme of the 7th Framework Programme for R\&D (FP7) for funding this research. The contents of this paper do not represent the opinion of the EU, which is not responsible for any use that might be made of them.

\section{References}

1. Healy, L. and Kynigos, C.: Charting the microworld territory over time: design and construction in mathematics education. ZDM, 42(1):63-76, (2010)

2. Noss, R. and Hoyles, C.: Windows on mathematical meanings: Learning cultures and computers. Dordrecht: Kluwer. (1996)

3. Scardamalia, M. \& Bereiter, C.: Computer support for knowledge-building communities. Journal of the learning sciences. 3(3). pp 265-283. (1993)

4. Stahl, G.: Group cognition: Computer support for building collaborative knowledge. Cambridge, MA: MIT Press. (2006)

5. Scheuer, O., Loll, F., Pinkwart, N. \& McLaren, B.M.: Computer-supported argumentation: A review of the state of the art. International Journal of Computer-Supported Collaborative Learning, 5(1), 43-102. (2010)

6. Tsovaltzi, D., Rummel, N., McLaren, B.M., Pinkwart, N., Scheuer, O., Harrer, A. and Braun, I.: Extending a virtual chemistry laboratory with a collaboration script to promote conceptual learning. International Journal of Technology Enhanced Learning, Vol. 2, Nos. 1/2,91-110. (2010) 
7. Dragon, T., Woolf, B.P., Murray, T.: Intelligent Coaching for Collaboration in Ill-Defined Domains. Poster paper, Proceedings of the 14th International Conference of Artificial Intelligence in Education, AIED 2009. Brighton, UK (2009)

8. Noss, R., Hoyles, C., Mavrikis, M., Geraniou, E., Gutierrez-Santos, S., and Pearce, D.: Broadening the sense of 'dynamic': a microworld to support students' mathematical generalisation. Special Issue of The International Journal on Mathematics Education (ZDM): Transforming Mathematics Education through the Use of Dynamic Mathematics Technologies, 41(4):493-503. (2009)

9. Mavrikis, M., Noss, R., Hoyles, C. Geraniou E.: Sowing the seeds of algebraic generalisation: designing epistemic affordances for an intelligent microworld. In Noss, R. and DiSessa, A. (eds) Special Issue on Knowledge Transformation, Design and Technology, Journal of Computer Assisted Learning. (to appear, 2011)

10. Loll, F., Pinkwart, N., Scheuer, O., \& McLaren, B.M.: Towards a flexible intelligent tutoring system for argumentation. In I. Adeo, N. Chen, Kinshuk, D. Sampson, \& L. Zaitseva (Eds.), Proceedings of the 9th IEEE International Conference on Advanced Learning Technologies (ICALT 2009) (p. 647-648). Los Alamitos (CA). (2009)

11. Scheuer, O., McLaren, B.M., Loll, F., \& Pinkwart, N.: An analysis and feedback infrastructure for argumentation learning systems. In V. Dimitrova, R. Mizoguchi, B. du Boulay, \& A. Graesser (Eds.), Proceedings of the 14th International Conference on Artificial Intelligence in Education (AIED-09), (pp. 629-631). IOS Press. (2009)

12. Gutierrez-Santos, S., Mavrikis, M. and Magoulas, G.: Layered Development and Evaluation for Intelligent Support in Exploratory Environments: The Case of Microworlds, Volume 6094 of Lecture Notes in Computer Science, pp. 105-114. Heidelberg: Springer Berlin. (2010)

13. Geraniou, G., Mavrikis, M., Hoyles, C.: Noss, R. Students' justification strategies on equivalence of quasi-algebraic expressions. International Conference on Psychology of Mathematics Education. Ancara, Turkey (2011)

14. Dragon, T., Floryan, M., Woolf, B.P., Murray, T.: Recognizing Dialogue Content in Student Collaborative Conversation. Proceedings of the 10th International Conference for Intelligent Tutoring Systems, ITS 2010. Pittsburgh, PA (2010)

15. McLaren, B.M., Scheuer, O., \& Mikšátko, J.: Supporting collaborative learning and eDiscussions using artificial intelligence techniques. International Journal of Artificial Intelligence in Education (IJAIED) 20(1), 1-46. (2010)

16. Pearce-Lazard, D., Poulovassilis, A. \& Geraniou, E.: The design of teacher assistance tools in an exploratory learning environment for mathematics generalisation. In: Proceedings of the $5^{\text {th }}$ European Conference on Technology Enhanced Learning (EC-TEL), Barcelona. Vol. 6383 of Springer Lecture Notes in Computer Science, pp. 260-275. (2010) 